\title{
Teaching Design in an Undergraduate Heat Transfer Course
}

\author{
A. Dolatabadi, R. Dhiman, S. Chandra \\ Department of Mechanical and Industrial Engineering \\ University of Toronto
}

\begin{abstract}
Third year undergraduate mechanical engineering students at the University of Toronto take a one-semester course in heat and mass transfer that is taught as a course in design of electronic cooling systems, combining theory with design and experiments. At the start of the course students are introduced to heat transfer problems faced by the electronics industry and cooling technologies. Heat transfer theory is then presented by analyzing electronic cooling systems. A combined numerical and experimental project is given to design a cooling system for an electronic instrument. Students are given a kit that includes a circuit board, heat sinks and a cooling fan. Components generating heat are represented by square aluminum plates clamped around thin heaters that can be placed anywhere on the circuit board. Students write a computer code to solve heat transfer equations and predict temperature distributions in the circuit board. The accuracy of these predictions is verified by experimental measurements. Results are submitted in the form of a report written from the perspective of a thermal design engineer working in a company that manufactures electronic equipment.
\end{abstract}

\section{Introduction}

Third year undergraduate mechanical engineering students at the University of Toronto take a onesemester course in heat and mass transfer. The weekly teaching schedule for the course includes three onehour lectures, a tutorial and a laboratory session. Traditionally this course has been taught with an emphasis on mathematical fundamentals, including formulation and solution of mass, momentum and energy conservation equations. Laboratories were highly structured, with students given detailed instructions of the measurements and calculations to be done. A design project, typically completed in the last month of the session, was independent of the laboratories.

Feedback from students showed that they viewed the course as useful though not very exciting. The laboratories received much criticism as being routine and leaving little room for student initiative. In light of these evaluations it was decided to revise the course extensively, teaching it as a design course that combined theory with a design project that had both analytical and experimental components. The aims of the course were to:

a) Provide a thorough grounding in heat and mass transfer theory.

b) Teach design of heat transfer systems.

c) Teach numerical solution of heat transfer problems.

d) Give an appreciation of how theory is applied to practical engineering problems.

e) Offer practice in teamwork and report writing.

f) Give hands-on experience in building a cooling system and making temperature measurements.

This paper will describe the structure of the course after it was reorganized to address the goals listed above.

\section{Course Structure}

Heat and mass transfer is taught as a course in design of electronic cooling systems. Several other industrial applications could have been chosen. Electronic cooling was selected because: a) it is currently an active, important and interesting area of research; and b) all students are familiar with cooling systems in personal computers and other electronic devices, and can easily visualize the type of equipment discussed in class in a way that they might not be able to do with other industrial machinery. The textbook by 
Cengel [1] contains a chapter discussing electronic cooling. Though this chapter is placed at the end of the book it can be taught without covering the preceding theory, since it is largely descriptive and lists empirical heat transfer correlations of the type found in datasheets for commercially available cooling equipment such as heat sinks and cooling fans. Within the first week of the course students are asked to use such correlations, without understanding how they were derived, to design cooling systems for different electronic units. They are asked to obtain specification sheets for cooling components from manufacturers. This approach very quickly makes them familiar with the application of heat transfer. Heat transfer theory is subsequently presented to the class as a way of explaining how correlations given in datasheets are obtained. Emphasis is placed not just on mathematical rigor but also on gaining a good physical understanding of dimensionless parameters that are used to correlate heat transfer data.

\section{Numerical model}

A major portion of the course is based on a combined numerical and experimental project. The objective is to design the cooling system for an electronic instrument that contains several chips emitting heat. Students work in teams of three to four to prepare a design in which they select the location of chips on a circuit board and choose heat sinks and a cooling fan that will keep the maximum temperature on the circuit board below a prescribed value. They are also required to keep the chips as close as possible to each other to minimize electrical interference. The design project has two parts. The first part is analytical, in which students calculate temperature distributions in the circuit board for different positions of the chips. They solve the two-dimensional, steadystate heat conduction equations in the circuit board by using a finite-difference approximation and write a computer code to solve the equations numerically. The exercise is useful in teaching students how commercially available computer codes work and what their limitations are.

\section{Experimental Verification}

Students perform experiments to test the accuracy of their calculations. Each team is given a kit that includes a circuit board, two heat sinks and a cooling fan (see figure 1).

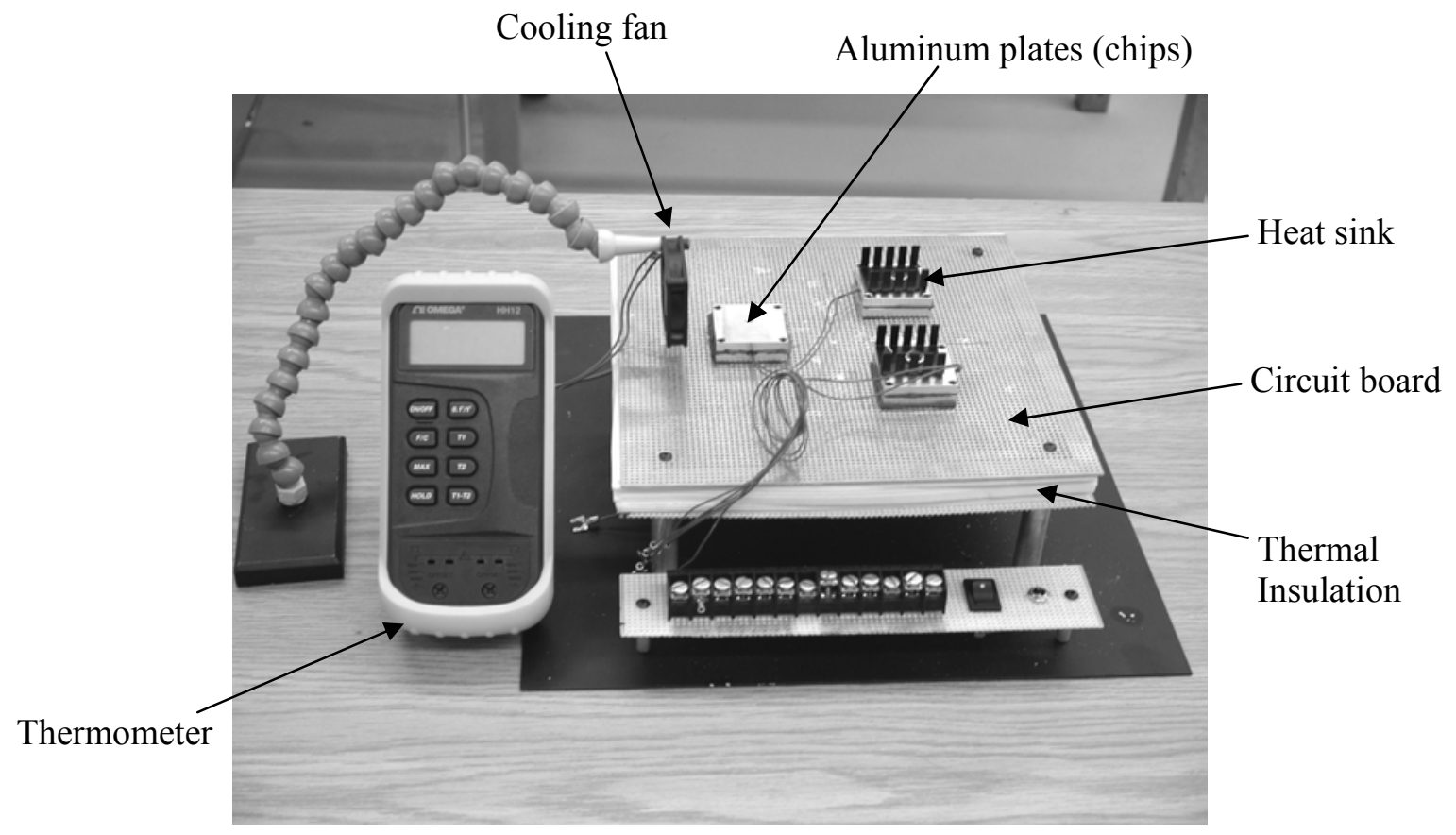

Figure 1. A photograph of experimental apparatus. 
The circuit board is an electrical prototyping board made of epoxy glass with copper cladding on one side to improve heat dissipation. It has rows of holes spaced $2.5 \mathrm{~mm}$ apart drilled through its entire surface. The bottom of the board is insulated to minimize heat loss. Electronic components generating heat are represented by square aluminum plates $(35 \mathrm{x}$ $35 \times 5 \mathrm{~mm}$ in size) clamped around thin Kapton heaters, each dissipating about $7 \mathrm{~W}$ (see figure 2). To minimize thermal contact resistance between the surface of the Kapton heater and aluminum plates, high-thermal conductivity interface pads are used. Each chip is secured in place with the help of screws passing through the circuit board holes, and can be positioned at any desired location on the circuit board.

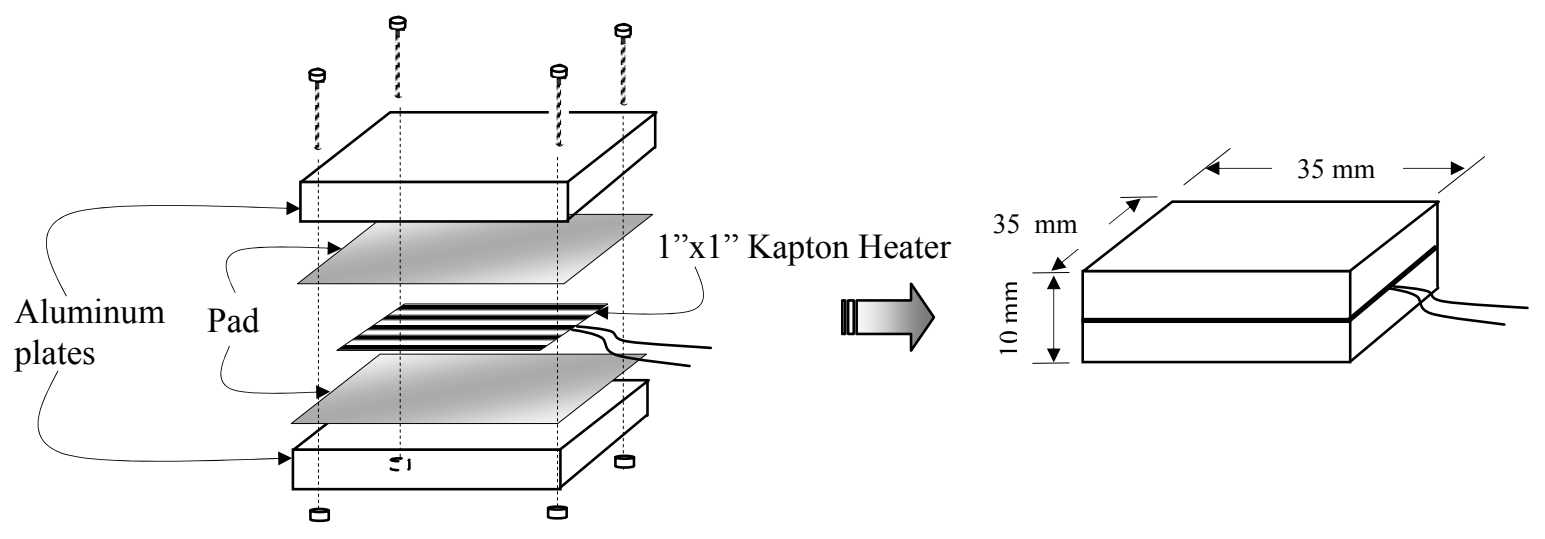

Figure 2. Simulated chip and its components.

Two heat sinks and one cooling fan are included in each kit. The heat sinks are commercially available models made of aluminum with a black anodized finish. The heat sink is clamped on the top surface of the chip with a high-thermal conductivity pad in between to reduce the thermal contact resistance. A cooling fan is provided that can be placed anywhere on the circuit board, at any desired angle. A dualinput, digital thermometer along with K-type (Chromel-Alumel) thermocouples is used to measure the temperature at any desired location on the circuit board. Each kit is powered by a $24 \mathrm{~V}, 2.1 \mathrm{~A}$ power supply.

Students are given relatively few instructions about what measurements are to be made or how best to arrange the chips and other cooling components. They are required to note discrepancies between their calculated value and measurements and either correct their analysis, repeat measurements, or explain why a difference exists.

Once the team is confident of the accuracy of its computer model they can use it to explore other possible layouts of components on the circuit board and select one that meets design requirements. They are encouraged to produce the simplest design possible, using the fewest components, while remaining below the specified limits for circuit board temperature.

Results are submitted in the form of a report written from the perspective of a thermal design engineer working in a company that manufactures electronic equipment. They are given a standard format for the report so that it includes an executive summary, introduction, discussion of results, and recommendations. The report is evaluated for both technical content and writing style.

\section{Conclusions}

The heat and mass transfer course taught to third year undergraduate students in mechanical engineering at the University of Toronto is taught as a course in electronic cooling, of which a large component is a design project that includes both numerical analysis and experimental work. The course gives students an appreciation of how theory is applied, how heat transfer systems are designed, and the difficulties in matching theoretical predictions with experimental measurements. Feedback from students has shown that 
it is an effective way of engaging the interest of students and of demonstrating engineering practice. It also is a useful way of encouraging teamwork and enhancing communication skills.

\section{References}

[1] Cengel, Yunus A., Heat Transfer: A practical approach, $2^{\text {nd }}$ edition, McGraw Hill publishing company, New York, 2003. 